\title{
Impact of Sarcopenia in Patients with Unresectable Locally Advanced Esophageal Cancer Receiving Chemoradiotherapy
}

\author{
SHO SATO $^{1}$, CHIKARA KUNISAKI ${ }^{1}$, HIDEAKI SUEMATSU ${ }^{1}$, YUSAKU TANAKA ${ }^{1}$, \\ HIROSHI MIYAMOTO ${ }^{1}$, TAKASHI KOSAKA ${ }^{1}$, NORIO YUKAWA ${ }^{1}$, \\ KUNIYA TANAKA $^{1}$, KEI SATO ${ }^{1}$, HIROTOSHI AKIYAMA ${ }^{2}$ and ITARU ENDO ${ }^{2}$ \\ ${ }^{1}$ Department of Surgery, Gastroenterological Center, Yokohama City University, Yokohama, Japan; \\ ${ }^{2}$ Department of Gastroenterological Surgery, \\ Yokohama City University Graduate School of Medicine, Yokohama, Japan
}

\begin{abstract}
Background: Esophageal cancer often involves direct invasion of adjacent organs and patient survival rates are low. Sarcopenia has been reported to be associated with a poor prognosis in several types of malignancies. However, the impact of sarcopenia on the long-term survival of patients with unresectable locally advanced esophageal cancer remains unclear. Patients and Methods: A total of 48 patients undergoing definitive chemoradiotherapy at our Institution from October 2012 to December 2015 were enrolled; their data were compared according to patient skeletal muscle index (SMI): low SMI (sarcopenia group), $n=34$; normal SMI (non-sarcopenia group), $n=14$. Results: There were no significant differences in the incidence of severe adverse events and dose reduction rate between the two groups. The incidence of nutritional support was significantly higher in the groups with sarcopenia than in the non-sarcopenia group $(44.1 \%$ vs. $7.1 \%, p=0.077)$. Response rates were significantly lower in the sarcopenia group than in the non-sarcopenia group $(43.8 \%$ vs. $78.6 \%, p=0.025)$. The overall survival rate in the group with sarcopenia was significantly lower than that in the non-sarcopenia group (3year: $36.95 \%$ vs. $63.9 \%, p=0.018)$. Conclusion: Sarcopenia prior to treatment may worsen the long-term survival of patients with unresectable locally advanced esophageal cancer. Further well-designed prospective trials are needed to estimate whether adequate nutritional support has a favorable impact on therapeutic outcomes in this population.
\end{abstract}

This article is freely accessible online.

Correspondence to: Chikara Kunisaki, MD, Ph.D., 4-57 Urafunecho, Minami-ku, Yokohama, 232-0024, Japan. Tel: +81 452615656, Fax: +81 452619492, e-mail: s0714@med.yokohama-cu.ac.jp

Key Words: Sarcopenia, esophageal cancer, prognosis, nutritional support.
Esophageal cancer is the eighth most common cancer in the world and a growing epidemic, with approximately 460,000 new diagnoses, and resulting in 380,000 deaths worldwide annually $(1,2)$. The prognosis of esophageal cancer remains poor with a 5-year overall survival rate of less than $20 \%$ (3, 4). Although curative surgery is a treatment for patients with resectable esophageal cancer, approximately $50 \%$ of patients have unresectable locally advanced tumor invading the adjacent organs or radiographically visible metastases (5). The standard treatment for unresectable locally advanced esophageal cancer is definitive chemoradiotherapy (6), but survival rates remain low.

Currently, sarcopenia, that is defined as the severe depletion of skeletal muscle mass and strength, is considered the most relevant phenotype of cachexia and has been linked to poor prognosis in several types of cancer (7-9). With respect to patients with esophageal cancer, it has been reported that sarcopenia is related to dose-limiting toxicity during neo-adjuvant chemotherapy and high anastomotic leakage rates $(7,10)$.

However, the impact of sarcopenia prior to treatment on the long-term prognosis of patients with unresectable locally advanced esophageal cancer remains unclear. Therefore, this retrospective single-institution study aimed to evaluate the impact of pretreatment sarcopenia as a prognostic factor in patients with unresectable locally advanced esophageal cancer receiving definitive chemoradiotherapy.

\section{Patients and Methods}

Patients. From October 2012 to December 2015, a series of 48 patients diagnosed with unresectable locally advanced esophageal cancer underwent definitive chemoradiotherapy at the Department of Surgery, Gastroenterological Center, Yokohama City University and its related institution. For all patients, tumors were confirmed histologically as squamous cell carcinoma. This retrospective study protocol conformed to the provisions of the Declaration of Helsinki and was approved by the Institutional Review Board for the Use of 
Human Subjects at the Yokohama City University School of Medicine (approval number: B180100023).

Chemoradiotherapy. From October 2012, patients with unresectable esophageal cancer initially underwent therapy with 5-fluorouracil plus cisplatin with radiotherapy. Patients under the age of 75 years were treated with concurrent chemoradiotherapy and patients over the age of 75 years were treated with sequential chemoradiotherapy tin order to avoid severe adverse events. Cisplatin was administered at a dose of $70 \mathrm{mg} / \mathrm{m}^{2}$ by slow drip infusion on days 1 and 29.5 -fluorouracil was administered at a dose of $700 \mathrm{mg} / \mathrm{m}^{2}$ per day by continuous infusion for $24 \mathrm{~h}$ on days 1-4 and days 29-32 in concurrent chemoradiotherapy or days 1-5 and days 29-33 in sequential chemoradiotherapy. Two liters of hydration was administered by continuous infusion for $24 \mathrm{~h}$ on days1-5 and days 29-33.

Radiation therapy was delivered with megavoltage equipment (6 MV) with anterior- and posterior-opposed fields up to $40 \mathrm{~Gy}$ to the primary tumor and regional lymph node area followed by a booster dose of $20 \mathrm{~Gy}$ to the primary tumor and locally enlarged lymph nodes using an oblique-opposed technique to exclude the spinal cord. Radiation therapy was performed 5 days per week at $2 \mathrm{~Gy} / \mathrm{day}$.

After initial chemoradiotherapy, patients without complete remission underwent docetaxel plus cisplatin therapy as a second-line chemotherapy. Docetaxel was administered at a dose of $60 \mathrm{mg} / \mathrm{m}^{2}$ on day 1 and cisplatin was administered at a dose of $60 \mathrm{mg} / \mathrm{m}^{2}$ on day 1. Two liters of hydration was administered by continuous infusion for $24 \mathrm{~h}$ on days 1-4. Each course lasted three weeks and was repeated until disease progression.

For third-line chemotherapy, a twice-daily dose of $40 \mathrm{mg} / \mathrm{m}^{2}$ S-1 was administered orally for four consecutive weeks. This was followed by a drug-free-interval of 2 weeks. The cycles of chemotherapy were repeated until obvious progression of disease.

For fourth-line chemotherapy, paclitaxel was administered at a dose of $100 \mathrm{mg} / \mathrm{m}^{2}$ on days $1,8,15,22,29$, and 36 . This was followed by a drug-free-interval of two weeks. Each course was repeated until progression of disease or until the patient refused treatment.

During the treatment, blood tests were carried out at least twice a week. Chemoradiotherapy or chemotherapy was discontinued if grade 3 or 4 adverse events determined by Common Terminology Criteria Events (CTCAE) version 4.0 (11) occurred, but resumed when symptoms improved. The tumor response was assessed using the Response Evaluation Criteria in Solid Tumors Guideline (RECIST version 1.1) 1 month after chemoradiotherapy (12). When a tumor showed a complete response (CR), two additional courses of the same chemotherapy were performed and the patients were followed up in the short-term with an esophagogastroduodenoscopy and CT scan every 3-6 months.

Skeletal muscle tissue measurement. Skeletal muscle tissue areas were measured by the SYNAPSE VINCENT system (Fuji Film Co. Ltd., Tokyo, Japan). Computed tomographic scans taken before and after chemoradiotherapy were evaluated. The area covered by skeletal muscle was calculated from pixels in the density range of -29 to +150 Hounsfield units (HUs). Two adjacent axial images within the same series at the level of the third lumbar vertebrae (L3) in the inferior direction were selected for analysis of the total muscle cross-sectional area $\left(\mathrm{cm}^{2}\right)$ and averaged for each patient. The muscle area normalized by the square of the patient's height $\left(\mathrm{m}^{2}\right)$ was defined as the skeletal muscle index (SMI) $\left(\mathrm{cm}^{2} / \mathrm{m}^{2}\right)(9)$. Pre-defined cut-offs for sarcopenia $\left(\leq 52.4 \mathrm{~cm}^{2} / \mathrm{m}^{2}\right.$ for men and $\leq 38.5 \mathrm{~cm}^{2} / \mathrm{m}^{2}$ for women) were used to define sarcopenia $(10,13)$ and patients were subsequently divided into groups with and without saropenia. This index was calculated on the day before chemoradiotherapy.

Nutritional support. Amounts of oral intake energy (OIE) and nutritionally supported energy (NSE) were calculated from medical records over a 1-week hospitalization period for first-line chemoradiotherapy. First, total energy expenditure (TEE) of all patients was calculated using the Harris-Benedict equation and the activity factor was calculated as 1.2. Nutritional support was administered for the patients with poor oral intake (OIE/TEE <0.6) by administration of semi-digestive state nutrients for patients without obstruction by tumor, and elemental diet via enteral feeding tube for patients with obstruction by tumor. Nutritional support was administered before the start of chemoradiotherapy, and continued throughout the whole period of chemoradiotherapy.

Patient data. Clinical data for all cases were collected from the prospectively maintained database at our Institution. The pathological classification was based on the esophageal cancer TNM (tumor-node metastasis) staging system of the Union for International Cancer Control (eighth edition) (14). Glasgow Prognostic Scores (GPSs) were assessed from blood tests carried out the day before chemoradiotherapy started. Patients with serum C-reactive protein $>10 \mathrm{mg} / \mathrm{l}$ and hypoalbuminemia $(<35 \mathrm{~g} / \mathrm{l})$ were given a score of 2 , those with only one of these biochemical abnormalities a score of 1 , and those with neither of these abnormalities a scored of 0 (15). Neutrophil lymphocyte ratio (16) and prognostic nutritional index (PNI) (17) were also calculated on the day before chemoradiotherapy.

Statistical analysis. Continuous data are expressed as the median (range). Data of different groups were compared using Wilcoxon test. Categorical data were analyzed using chi-square test. Overall survival rates from the start of treatment were calculated according to Kaplan-Meier method, and differences between groups were tested for significance using the log-rank test. Multivariate survival analysis and the calculation of hazard ratios used a model of Cox proportional hazards regression including covariates that gave values of $p<0.10$ in univariate survival analysis. All statistical analyses were performed using JMP ${ }^{\circledR} 13$ (SAS Institute Inc., Cary, NC, USA). Differences with probability values of $p<0.05$ were considered as significant.

\section{Results}

Patient characteristics. Patient characteristics in the sarcopenia group $(n=34)$ and the non-sarcopenia group $(n=14)$ are shown in Table I. A total of 34 patients $(70.8 \%)$ had invasion of the trachea or left main bronchus, nine patients $(18.8 \%)$ had invasion of the aorta and 10 patients $(20.8 \%)$ had invasion of other areas (recurrent nerve, carotid artery, lung, and vertebra) which were considered unresectable invaded organs. No patient had distant metastasis, and tumor in every patient was diagnosed as stage IIIC in the TNM classification (14).

There were no significant differences in patient characteristics other than body mass index (BMI). The BMI 
Table I. Patient characteristics of both sarcopenia and non-sarcopenia groups.

\begin{tabular}{|c|c|c|c|}
\hline Variable & Sarcopenia group $(n=34)$ & Non-sarcopenia group $(n=14)$ & $p$-Value \\
\hline Gender: M/F, n (\%) & $23(67.7 \%) / 11(32.4 \%)$ & $9(64.3 \%) / 5(35.7 \%)$ & 0.823 \\
\hline Age (years) & $65.5(41-79)$ & $70.0(53-77)$ & 0.097 \\
\hline \multicolumn{4}{|l|}{ Comorbidity, n (\%) } \\
\hline Cardiovascular disease & $2(5.9 \%)$ & $0(0 \%)$ & 0.234 \\
\hline Pulmonary disease & $2(5.9 \%)$ & $0(0 \%)$ & 0.234 \\
\hline Diabetes mellitus & $3(8.8 \%)$ & $1(7.1 \%)$ & 0.846 \\
\hline PNI* & $48.8(36.5-59.2)$ & $49.7(43.8-55.9)$ & 0.919 \\
\hline BMI $\left(\mathrm{kg} / \mathrm{m}^{2}\right)$ & $18.4(13.3-25.0)$ & $22.8(17.8-27.4)$ & 0.001 \\
\hline $\operatorname{BSA}\left(\mathrm{m}^{2}\right)$ & $1.77(1.30-2.13)$ & $1.70(1.29-2.04)$ & 0.439 \\
\hline GPS, n (\%) & & & 0.963 \\
\hline 0 & $23(67.7 \%)$ & $10(71.4 \%)$ & \\
\hline 1 & $8(23.5 \%)$ & $3(21.4 \%)$ & \\
\hline 2 & $3(8.8 \%)$ & $1(7.1 \%)$ & \\
\hline $\mathrm{CRP}(\mathrm{mg} / \mathrm{dl}) *$ & $0.373(0.024-8.677)$ & $0.628(0.033-3.527)$ & 0.786 \\
\hline $\mathrm{Alb}(\mathrm{mg} / \mathrm{dl})^{*}$ & $4.15(3.1-4.8)$ & $4.15(3.4-4.6)$ & 0.665 \\
\hline NLR* & $3.31(1.41-15.9)$ & $3.13(1.14-7.05)$ & 0.461 \\
\hline Tumor size $(\mathrm{mm})^{*}$ & $60.0(30-120)$ & $60.0(20-92)$ & 1.000 \\
\hline \multicolumn{4}{|l|}{ Invaded organ, $\mathrm{n}(\%)$} \\
\hline Trachea/main bronchus & $23(67.6 \%)$ & $12(85.7 \%)$ & 0.182 \\
\hline Aorta & $6(17.6 \%)$ & $3(21.4 \%)$ & 0.767 \\
\hline Other & $8(23.5 \%)$ & $2(14.3 \%)$ & 0.461 \\
\hline Lymph node metastasis, $\mathrm{n}(\%)$ & $12(85.7 \%)$ & $31(91.2 \%)$ & 0.583 \\
\hline $\mathrm{SCC}(\mathrm{ng} / \mathrm{ml}) *$ & $1.65(0.6-15.1)$ & $1.3(0.6-10.6)$ & 0.615 \\
\hline
\end{tabular}

PNI: Prognostic nutritional index, GPS: Glasgow prognostic score, NLR: neutrophil:lymphocyte ratio, BMI: body mass index, BSA: body surface area, CRP: C-reactive protein, Alb: albumin, SCC: squamous cell carcinoma *Data are median (range).

Table II. Adverse effects, response to chemoradiotherapy and administration of subsequent chemotherapy of both the sarcopenia and the nonsarcopenia groups.

\begin{tabular}{lccc}
\hline Variable & Sarcopenia group (n=34) & Non-sarcopenia group (n=14) & $p$-Value \\
\hline Grade 3 or 4 AE, n (\%) & $5(14.7 \%)$ & $3(21.4 \%)$ & 0.577 \\
Dose reduction, n (\%) & $9(26.5 \%)$ & $6(42.9 \%)$ & 0.273 \\
Discontinuation, n (\%) & $4(11.8 \%)$ & $0(0 \%)$ & 0.088 \\
RT dose (Gy)* & $59.4(19.8-68.4)$ & $59.4(50.4-61)$ & 0.945 \\
Response, n (\%) & $3(8.8 \%)$ & $4(28.6 \%)$ & 0.092 \\
CR & $14(43.8 \%)$ & $11(78.6 \%)$ & 0.025 \\
CR + PR & & & \\
Subsequent chemotherapy & $19(55.9 \%)$ & $10(71.4 \%)$ & 0.310 \\
2nd line & $7(20.6 \%)$ & $5(35.7 \%)$ & 0.271 \\
3rd line & $4(11.8 \%)$ & $1(7.1 \%)$ & 0.633 \\
4th line & &
\end{tabular}

AE: Adverse effect. PR: partial response. CR: complete response. RT: radiotherapy. *Data are median (range).

was lower in the group with sarcopenia than in the group without [18.4 (13.3-25.0) versus $22.8(17.8-27.4) \mathrm{kg} / \mathrm{m}^{2}$, $p=0.001]$. There were no differences in tumor factors between the two groups.

Response to chemoradiotherapy and adverse events. There were no significant differences in the incidence of severe adverse events or the dose reduction rate between the two groups (Table II). Discontinuation of chemoradiotherapy tended to be more frequent in the group with sarcopenia ( $11.8 \%$ versus $0 \%, p=0.088$ ). Reasons for discontinuation were perforation of aorta, severe diarrhea and neutropenia, cancer death during chemoradiotherapy, and drug allergy in one case each in the sarcopenia group. Response rates 
Table III. Nutritious status of the sarcopenia and non-sarcopenia groups.

\begin{tabular}{lccc}
\hline Variable & Sarcopenia group $(\mathrm{n}=34)$ & Non-sarcopenia group $(\mathrm{n}=14)$ & $p$-Value \\
\hline Number of patients receiving nutritional support, $\mathrm{n}(\%)$ & $15(44.1 \%)$ & $1(7.1 \%)$ & 0.007 \\
Nutritionally supported energy (kcal) (mean \pm SD) & $564.4 \pm 789.0$ & $107.1 \pm 400.9$ & 0.017 \\
Total intake energy (kcal) (mean \pm SD) & $1513.6 \pm 367.1$ & $1430.1 \pm 380.1$ & 0.488 \\
Body weight $(\%) *$ & $98.9(86.8-113.6)$ & $98.7(88.2-107.0)$ & 0.696 \\
SMI $(\%)^{*}$ & $99.1(80.7-115.4)$ & $93.7(77.1-108.2)$ & 0.250 \\
\hline
\end{tabular}

SMI: Skeletal muscle index.*Data are median (range) difference between the start and end of chemoradiotherapy.

[complete response (CR) and partial response (PR)] were significantly lower in the sarcopenia group than in the nonsarcopenia group $(43.8 \%$ versus $78.6 \%, p=0.025)$. Moreover, the $\mathrm{CR}$ rate tended to be lower in the sarcopenia group $(8.8 \%$ versus $28.6 \%, p=0.092)$. After $\mathrm{CR}$ was confirmed, four patients (two patients in each group) experienced recurrence: two had local recurrence and two had distant metastasis. There were no differences in administration of follow-up chemotherapy between the two groups.

Nutritional support for both groups. As previously described, nutritional support was administered for patients with poor oral intake. Both the incidence of nutritional support and nutritionally supported energy administration was significantly higher in the sarcopenia group than the non-sarcopenia group. Total intake energy, and the proportion of both body weight change and SMI change during chemoradiotherapy were not significantly different (Table III).

Overall survival. Overall survival rates of both groups are shown in Figure 1. The overall survival rate was significantly worse in the group with sarcopenia (at 3 years: $36.95 \% v s$. $63.9 \%, p=0.018$ ).

Prognostic factors for overall survival. Univariate analysis for overall survival showed that male gender, discontinuation of chemoradiotherapy, being a non-responder, and having sarcopenia were significantly associated with poor overall survival (Table IV).

The Cox proportional hazard regression model for overall survival showed that being male and being a responder were independent prognostic factors for poor overall survival in these patients. However, sarcopenia was rejected as an independent prognostic factor (Table V).

\section{Discussion}

This retrospective study showed that patients with unresectable locally advanced esophageal cancer with sarcopenia had worse responses to chemoradiotherapy and



Figure 1. Overall survival in patients with and without sarcopenia with unresectable locally advanced esophageal cancer treated with definitive chemoradiotherapy. The difference in overall survival between the sarcopenia group $(n=34)$ and the non-sarcopenia group $(n=14)$ was significant (36.95\% vs. 63.9\%, $p=0.018$ ).

poorer overall survival than the those without sarcopenia. Although patients with sarcopenia received nutritional support to an equivalent level to patients without sarcopenia, this support appears to have had no effect.

Recently, data obtained from various large databases have linked sarcopenia to a poor prognosis in patients with gastrointestinal cancer (9). In addition, there is significant evidence showing that sarcopenia is independently associated with poor response to cancer therapy in pancreatic (18), breast (19), colorectal $(13,20)$, and renal-cell (21), and hepatic (22) cancer. Sarcopenia carries a high risk of morbidity (23-26) and confers poor long-term survival after resection of esophageal cancer (27-29). However, there is very little evidence showing the correlation between sarcopenia and long-term outcomes in patients with unresectable esophageal cancer undergoing chemoradiotherapy. Harada et al. reported that patients with esophageal cancer without lymph involvement had poor longterm outcomes, however, their study included both 
Table IV. Univariate analysis for overall survival.

\begin{tabular}{|c|c|c|c|c|c|c|}
\hline Variable & $\mathrm{N}$ & 3-Year survival $(\%)$ & MST (months) & HR & $95 \% \mathrm{CI}$ & $p$-Value \\
\hline \multicolumn{7}{|l|}{ Gender } \\
\hline Female & 16 & 70.0 & NR & 1 & & \\
\hline Male & 32 & 35.0 & 16.8 & 3.691 & $1.498-10.47$ & 0.0039 \\
\hline \multicolumn{7}{|l|}{ Adverse effect } \\
\hline Grade 1,2 & 40 & 40.9 & 17.8 & 1 & & \\
\hline Grade 3,4 & 8 & 62.5 & NR & 0.659 & $0.155-1.936$ & 0.4816 \\
\hline \multicolumn{7}{|c|}{ Dose reduction } \\
\hline Yes & 15 & 57.5 & NR & 1 & & \\
\hline No & 33 & 16.8 & 39.4 & 1.920 & $0.764-5.825$ & 0.1732 \\
\hline \multicolumn{7}{|c|}{ Discontinuation of CRT } \\
\hline Yes & 4 & 0 & 4.0 & 5.873 & & \\
\hline No & 44 & 47.9 & 24.8 & 1 & $1.329-18.59$ & 0.0234 \\
\hline \multicolumn{7}{|l|}{$\mathrm{CR}$} \\
\hline Yes & 7 & 64.3 & NR & 0.383 & & \\
\hline No & 41 & 42.1 & 16.8 & 1 & $0.061-1.313$ & 0.1408 \\
\hline \multicolumn{7}{|l|}{$\mathrm{PR} / \mathrm{CR}$} \\
\hline Yes & 25 & 60.2 & 60.2 & 0.389 & & \\
\hline No & 21 & 24.4 & 24.4 & 1 & $0.161-0.919$ & 0.0317 \\
\hline \multicolumn{7}{|l|}{ SCC } \\
\hline$\geq 1.5$ & 22 & 35.5 & 17.8 & 1.596 & & \\
\hline$<1.5$ & 21 & 58.9 & 39.2 & 1 & $0.250-1.528$ & 0.3022 \\
\hline \multicolumn{7}{|l|}{ GPS } \\
\hline 0 & 33 & 46.9 & 24.8 & 1 & & \\
\hline 1 or 2 & 15 & 42.4 & 20.2 & 1.264 & $0.483-2.986$ & 0.6136 \\
\hline \multicolumn{7}{|l|}{ NLR } \\
\hline$\geq 2.5$ & 34 & 41.2 & 24.8 & 1.246 & & \\
\hline$<2.5$ & 14 & 46.7 & 20.2 & 1 & $0.516-3.463$ & 0.6384 \\
\hline \multicolumn{7}{|l|}{ PNI } \\
\hline$<40$ & 3 & 50.0 & 20.2 & 0.668 & & \\
\hline$\geq 40$ & 45 & 45.0 & NR & 1 & $0.037-3.202$ & 0.6756 \\
\hline \multicolumn{7}{|l|}{ Sarcopenia } \\
\hline Yes & 34 & 37.0 & 15.2 & 3.474 & & \\
\hline No & 14 & 63.9 & NR & 1 & $1.283-12.17$ & 0.0125 \\
\hline \multicolumn{7}{|l|}{ BMI } \\
\hline$<20$ & 27 & 43.5 & 12.2 & 1.741 & & \\
\hline$\geq 20$ & 11 & 49.2 & 24.8 & 1 & $0.759-4.198$ & 0.1913 \\
\hline \multicolumn{7}{|c|}{ Muscle depletion during CRT } \\
\hline$\geq 10 \%$ & 12 & 32.1 & 17.8 & 1.310 & & \\
\hline$<10 \%$ & 36 & 49.5 & 20.2 & 1 & $0.472-3.159$ & 0.5788 \\
\hline
\end{tabular}

MST: Median survival, HR: hazard ratio, CI: confidence interval, NR: not reached (>50\% patients surviving at study end), GPS: Glasgow prognostic score, NLR: neutrophil:lymphocyte ratio, PNI: prognostic nutritional index, PR: partial response, CR: complete response, BMI: body mass index, SCC: squamous cell carcinoma.

chemoradiotherapy and esophageal resection cases (7). To our knowledge, our retrospective study is the first report that shows the impact of sarcopenia on patients with unresectable locally advanced esophageal cancer.

The mechanisms by which skeletal muscle depletion shortens the survival of patients with malignant cancer remain unclear. In the current study, the response rates were worse in the sarcopenia group than the non-sarcopenia group, which indicates that tumors in the sarcopenia group had poorer sensitivity to cheoradiotherapy despite nutritional support. In addition, a recent study has putatively linked skeletal muscle depletion to molecular phenotypic changes in factors such as tumor necrosis factor alpha, interleukin-6, and insulin-like growth factor 1 (7). Sarcopenia is considered the most relevant phenotypic feature of cancer cachexia, and thus might reflect the high malignancy of advanced esophageal cancer and lead to poor long-term results $(7,30)$. In addition, sarcopenia is a prominent feature of malnutrition due to cancer progression. Undernutrition has been reported to be as frequent as $79 \%$ in patients with advanced esophageal cancer before starting treatment (31). In these patients, anorexia and dysphagia are the main factors 
Table V. Multivariate analysis for overall survival.

\begin{tabular}{lccc}
\hline Variable & HR & $95 \%$ CI & $p$-Value \\
\hline $\begin{array}{l}\text { Gender } \\
\text { Male }\end{array}$ & 4.293 & $1.446-15.92$ & 0.0075 \\
$\quad$ Female & 1 & & \\
$\begin{array}{l}\text { Discontinuation of CRT } \\
\text { Yes }\end{array}$ & 3.249 & $0.476-13.60$ & 0.1954 \\
$\quad$ No & 1 & & \\
PR/CR & & & \\
$\quad$ Yes & 0.253 & $0.096-0.656$ & 0.0050 \\
$\quad$ No & 1 & & \\
Sarcopenia \\
$\quad$ Yes \\
No
\end{tabular}

HR: Hazard ratio, CI: 95\% confidence interval, PR: partial response, CR: complete response, CRT: chemoradiotherapy.

involved in the onset of undernutrition (32). Basically, limitation of oral intake can be caused by tumor obstruction. Undernutrition has been reported as a factor predictive of treatment discontinuation and poor outcomes in patients treated at the palliative stage (33-35). In the current study, there was no significant difference in nutritional status measurements, such as PNI and albumin level between groups. Sarcopenia might be an independent nutritional indicator which affects the long-term prognosis in patients with unresectable esophageal cancer.

A previous study reported an association between sarcopenic obesity and dose-limiting toxicity during neoadjuvant chemotherapy for esophageal cancer (10). Moreover, sarcopenia was reported to be associated with a high risk of adverse events during chemotherapy for metastatic breast and colorectal carcinoma $(19,20,36)$. The reason for the frequent adverse events was that in a patient with a small lean compartment, a high drug dose is distributed in a small volume. Recent studies have shown that skeletal muscle volume decreases after neoadjuvant chemotherapy $(37,38)$, and $43.6 \%$ of patients show more than $10 \%$ of body weight loss during definitive chemoradiotherapy for advanced esophageal cancer (39). Patients undergoing chemotherapy for gastrointestinal cancer who experience weight loss usually suffer more frequent and severe toxicities (33). In our study, patients with sarcopenia had a lower BMI combined with weight loss before chemotherapy. However, there was no significant difference in the incidence of severe toxicities or dose reduction between groups. Patients in the sarcopenia group received nutritional support appropriately and there were no differences in total energy intake, body weight loss, and SMI change between the two groups. Therefore, positive nutritional support may prevent severe weight loss, muscle depletion, and severe adverse events in patients with unresectable esophageal cancer who cannot maintain sufficient oral intake. As a result, pretreatment sarcopenia lost its role as an independent prognostic factor for overall survival in this population.

There were some limitations to this study. Firstly, it was a retrospective study. Secondly, nutritional support was administered to $44.1 \%$ patients in the sarcopenia group; however, the usefulness of nutritional support for patients with sarcopenia is still unclear. Moreover, the volume of nutritional support required to achieve adequacy is unknown. It is necessary to evaluate skeletal muscle depletion routinely and to establish adequate nutritional support for patients with unresectable esophageal cancer before chemoradiotherapy. Thirdly, this was a single-institution study and the number of patients was small. Therefore, a multi-institutional study with more patients should be conducted.

In conclusion, sarcopenia may worsen the long-term prognosis of patients with unresectable locally advanced esophageal cancer. It is necessary to conduct a well-designed prospective trial to determine whether adequate nutritional support has a favorable impact on therapeutic outcomes in this population.

\section{Conflicts of Interest}

None.

\section{References}

1 Jemal A, Siegel R, Ward E, Hao Y, Xu J and Thun MJ: Cancer statistics, 2009. CA Cancer J Clin 59(4): 225-249, 2009.

2 Kamangar F, Dores GM and Anderson WF: Patterns of cancer incidence, mortality, and prevalence across five continents: defining priorities to reduce cancer disparities in different geographic regions of the world. J Clin Oncol 24(14): 21372150, 2006.

3 Di Fiore F, Lecleire S, Rigal O, Galais MP, Ben Soussan E, David I, Paillot B, Jacob JH and Michel P: Predictive factors of survival in patients treated with definitive chemoradiotherapy for squamous cell esophageal carcinoma. World J Gastroenterol 12(26): 4185-4190, 2006.

4 Keighley MR: Gastrointestinal cancers in Europe. Aliment Pharmacol Ther 18(Suppl 3): 7-30, 2003.

5 Enzinger PC and Mayer RJ: Esophageal cancer. N Engl J Med 349(23): 2241-2252, 2003.

6 Satake H, Tahara M, Mochizuki S, Kato K, Hara H, Yokota T, Kiyota N, Kii T, Chin K, Zenda S, Kojima T, Bando H, Yamazaki T, Iwasa S, Honma Y, Hamauchi S, Tsushima T and Ohtsu A: A prospective, multicenter phase I/II study of induction chemotherapy with docetaxel, cisplatin and fluorouracil (DCF) followed by chemoradiotherapy in patients with unresectable locally advanced esophageal carcinoma. Cancer Chemother Pharmacol 78(1): 91-99, 2016.

7 Harada K, Ida S, Baba Y, Ishimoto T, Kosumi K, Tokunaga R, Izumi D, Ohuchi M, Nakamura K, Kiyozumi Y, Imamura Y, Iwatsuki M, Iwagami S, Miyamoto Y, Sakamoto Y, Yoshida N, 
Watanabe $\mathrm{M}$ and Baba H: Prognostic and clinical impact of sarcopenia in esophageal squamous cell carcinoma. Dis Esophagus 29(6): 627-633, 2016.

8 Ishihara H, Kondo T, Omae K, Takagi T, Iizuka J, Kobayashi H, Hashimoto Y and Tanabe K: Sarcopenia predicts survival outcomes among patients with urothelial carcinoma of the upper urinary tract undergoing radical nephroureterectomy: a retrospective multiinstitution study. Int J Clin Oncol 22(1): 136-144, 2017.

9 Martin L, Birdsell L, Macdonald N, Reiman T, Clandinin MT, McCargar LJ, Murphy R, Ghosh S, Sawyer MB and Baracos VE: Cancer cachexia in the age of obesity: skeletal muscle depletion is a powerful prognostic factor, independent of body mass index. J Clin Oncol 31(12): 1539-1547, 2013.

10 Anandavadivelan P, Brismar TB, Nilsson M, Johar AM and Martin L: Sarcopenic obesity: A probable risk factor for dose limiting toxicity during neo-adjuvant chemotherapy in oesophageal cancer patients. Clin Nutr 35(3): 724-730, 2016.

11 Institute NC: Common Terminology Criteria for Adverse Events v4.0 NIH publication \# 09-7473, NCI, NIH, DHHS. May 29, 2009.

12 Therasse P, Arbuck SG, Eisenhauer EA, Wanders J, Kaplan RS, Rubinstein L, Verweij J, Van Glabbeke M, van Oosterom AT, Christian MC and Gwyther SG: New guidelines to evaluate the response to treatment in solid tumors. European Organization for Research and Treatment of Cancer, National Cancer Institute of the United States, National Cancer Institute of Canada. J Natl Cancer Inst 92(3): 205-216, 2000.

13 Prado CM, Baracos VE, McCargar LJ, Mourtzakis M, Mulder KE, Reiman T, Butts CA, Scarfe AG and Sawyer MB: Body composition as an independent determinant of 5-fluorouracilbased chemotherapy toxicity. Clin Cancer Res 13(11): 32643268, 2007.

14 Brierley JD, Gospodarowicz MK and Wittekind C (eds.): TNM Classification of Malignant Tumours, 8th Edition. Oxford, UK: Wiley-Blackwell; 2016.

15 Forrest LM, McMillan DC, McArdle CS, Angerson WJ and Dunlop DJ: Comparison of an inflammation-based prognostic score (GPS) with performance status (ECOG) in patients receiving platinum-based chemotherapy for inoperable nonsmall-cell lung cancer. Br J Cancer 90(9): 1704-1706, 2004.

16 Sharaiha RZ, Halazun KJ, Mirza F, Port JL, Lee PC, Neugut AI, Altorki NK and Abrams JA: Elevated preoperative neutrophil: lymphocyte ratio as a predictor of postoperative disease recurrence in esophageal cancer. Ann Surg Oncol 18(12): 33623369, 2011.

17 Onodera T, Goseki N and Kosaki G: Prognostic nutritional index in gastrointestinal surgery of malnourished cancer patients. Nihon Geka Gakkai Zasshi 85(9): 1001-1005, 1984.

18 Tan BH, Birdsell LA, Martin L, Baracos VE and Fearon KC: Sarcopenia in an overweight or obese patient is an adverse prognostic factor in pancreatic cancer. Clin Cancer Res 15(22): 6973-6979, 2009.

19 Prado CM, Baracos VE, McCargar LJ, Reiman T, Mourtzakis M, Tonkin K, Mackey JR, Koski S, Pituskin E and Sawyer MB: Sarcopenia as a determinant of chemotherapy toxicity and time to tumor progression in metastatic breast cancer patients receiving capecitabine treatment. Clin Cancer Res 15(8): 29202926, 2009.

20 Barret M, Antoun S, Dalban C, Malka D, Mansourbakht T, Zaanan A, Latko E and Taieb J: Sarcopenia is linked to treatment toxicity in patients with metastatic colorectal cancer. Nutr Cancer 66(4): 583-589, 2014.

21 Antoun S, Baracos VE, Birdsell L, Escudier B and Sawyer MB: Low body mass index and sarcopenia associated with doselimiting toxicity of sorafenib in patients with renal cell carcinoma. Ann Oncol 21(8): 1594-1598, 2010.

22 Harimoto N, Shirabe K, Yamashita YI, Ikegami T, Yoshizumi T, Soejima Y, Ikeda T, Maehara Y, Nishie A and Yamanaka T: Sarcopenia as a predictor of prognosis in patients following hepatectomy for hepatocellular carcinoma. Br J Surg 100(11): 1523-1530, 2013.

23 Ida S, Watanabe M, Yoshida N, Baba Y, Umezaki N, Harada K, Karashima R, Imamura Y, Iwagami S and Baba H: Sarcopenia is a predictor of postoperative respiratory complications in patients with esophageal cancer. Ann Surg Oncol 22(13): 44324437, 2015.

24 Makiura D, Ono R, Inoue J, Kashiwa M, Oshikiri T, Nakamura T, Kakeji Y, Sakai Y and Miura Y: Preoperative sarcopenia is a predictor of postoperative pulmonary complications in esophageal cancer following esophagectomy: A retrospective cohort study. J Geriatr Oncol 7(6): 430-436, 2016.

25 Nishigori T, Okabe H, Tanaka E, Tsunoda S, Hisamori S and Sakai Y: Sarcopenia as a predictor of pulmonary complications after esophagectomy for thoracic esophageal cancer. J Surg Oncol 113(6): 678-684, 2016.

26 Reisinger KW, Bosmans JW, Uittenbogaart M, Alsoumali A, Poeze M, Sosef MN and Derikx JP: Loss of skeletal muscle mass during neoadjuvant chemoradiotherapy predicts postoperative mortality in esophageal cancer surgery. Ann Surg Oncol 22(13): 4445-4452, 2015.

27 Nakashima Y, Saeki H, Nakanishi R, Sugiyama M, Kurashige J, Oki E and Maehara Y: Assessment of sarcopenia as a predictor of poor outcomes after esophagectomy in elderly patients with esophageal cancer. Ann Surg, 2017. doi: 10.1097/SLA.00000 00000002252. [Epub ahead of print]

28 Paireder M, Asari R, Kristo I, Rieder E, Tamandl D, BaSsalamah A and Schoppmann SF: Impact of sarcopenia on outcome in patients with esophageal resection following neoadjuvant chemotherapy for esophageal cancer. Eur J Surg Oncol 43(2): 478-484, 2017.

29 Sakai M, Sohda M, Miyazaki T, Yoshida T, Kumakura Y, Honjo H, Hara K, Ozawa D, Suzuki S, Tanaka N, Yokobori T and Kuwano H: Association of preoperative nutritional status with prognosis in patients with esophageal cancer undergoing salvage esophagectomy. Anticancer Res 38(2): 933-938, 2018.

30 Miyata H, Sugimura K, Motoori M, Fujiwara Y, Omori T, Yanagimoto Y, Ohue M, Yasui M, Miyoshi N, Tomokuni A, Akita H, Kobayashi S, Takahashi $\mathrm{H}$ and Yano M: Clinical assessment of sarcopenia and changes in body composition during neoadjuvant chemotherapy for esophageal cancer. Anticancer Res 37(6): 3053-3059, 2017.

31 Riccardi D and Allen K: Nutritional management of patients with esophageal and esophagogastric junction cancer. Cancer Control 6(1): 64-72, 1999.

32 Di Fiore F, Lecleire S, Pop D, Rigal O, Hamidou H, Paillot B, Ducrotte P, Lerebours E and Michel P: Baseline nutritional status is predictive of response to treatment and survival in patients treated by definitive chemoradiotherapy for a locally advanced esophageal cancer. Am J Gastroenterol 102(11): 25572563, 2007. 
33 Andreyev HJ, Norman AR, Oates J and Cunningham D: Why do patients with weight loss have a worse outcome when undergoing chemotherapy for gastrointestinal malignancies? Eur J Cancer 34(4): 503-509, 1998.

34 Chau I, Norman AR, Cunningham D, Waters JS, Oates J and Ross PJ: Multivariate prognostic factor analysis in locally advanced and metastatic esophago-gastric cancer - pooled analysis from three multicenter, randomized, controlled trials using individual patient data. J Clin Oncol 22(12): 2395-2403, 2004.

35 Crumley AB, McMillan DC, McKernan M, McDonald AC and Stuart RC: Evaluation of an inflammation-based prognostic score in patients with inoperable gastro-oesophageal cancer. $\mathrm{Br}$ J Cancer 94(5): 637-641, 2006.

36 Liu J, Motoyama S, Sato Y, Wakita A, Kawakita Y, Saito H and Minamiya Y: Decreased skeletal muscle mass after neoadjuvant therapy correlates with poor prognosis in patients with esophageal cancer. Anticancer Res 36(12): 6677-6685, 2016.

37 Awad S, Tan BH, Cui H, Bhalla A, Fearon KC, Parsons SL, Catton JA and Lobo DN: Marked changes in body composition following neoadjuvant chemotherapy for oesophagogastric cancer. Clin Nutr 31(1): 74-77, 2012.
38 Yip C, Goh V, Davies A, Gossage J, Mitchell-Hay R and Hynes O, Maisey N, Ross P, Gaya A, Landau DB, Cook GJ, Griffin N and Mason R: Assessment of sarcopenia and changes in body composition after neoadjuvant chemotherapy and associations with clinical outcomes in oesophageal cancer. Eur Radiol 24(5): 998-1005, 2014.

39 Di Fiore A, Lecleire S, Gangloff A, Rigal O, Benyoucef A, Blondin V, Sefrioui D, Quiesse M, Iwanicki-Caron I and Michel P, Di Fiore F: Impact of nutritional parameter variations during definitive chemoradiotherapy in locally advanced oesophageal cancer. Dig Liver Dis 46(3): 270-275, 2014.

Received January 5, 2018

Revised February 13, 2018

Accepted February 20, 2018 